\title{
Drug-Resistant Breast Cancer: Dwelling the Hippo Pathway to Manage the Treatment
}

\author{
Simran Kaur \\ Mohammad Zeeshan Najm ' \\ Mohammad Aasif Khan ${ }^{2}$ \\ Naseem Akhter (iD ${ }^{3}$ \\ Vyas M Shingatgeri ${ }^{I}$ \\ Mudra Sikenis' \\ Sadaf (iD ${ }^{4}$ \\ Abdulaziz A Aloliqi ${ }^{5}$ \\ 'School of Bioscience, Apeejay Stya \\ University, Gurugram, Haryana, India; \\ ${ }^{2}$ Department of Biosciences, Jamia Millia \\ Islamia University, New Delhi, India; \\ ${ }^{3}$ Department of Laboratory Medicine, \\ Faculty of Applied Medical Sciences, Al \\ Baha University, Al Baha, Saudi Arabia; \\ ${ }^{4}$ QuickisCool, Aitele Research LLP, \\ Sitamarhi, Bihar, India; ${ }^{5}$ Department of \\ Medical Biotechnology, College of \\ Applied Medical Sciences, Qassim \\ University, Buraydah, Saudi Arabia
}

\begin{abstract}
Breast cancer can be categorized as a commonly occurring cancer among women with a high mortality rate. Due to the repetitive treatment cycles, it has been noted that the patients develop resistance towards the chemotherapeutic drugs and remain unresponsive towards them. Therefore, many researchers are studying various signaling pathways involved in drug resistance for cancer treatment to overcome the obstacle. Hippo signaling is a widely studied pathway involved in tumor progression and controlling cell proliferation. Hence, understanding the aspects of the gene involved Hippo pathway would provide an insight into the mechanism behind the resistance and result in the development of new treatments. Here, we review the Hippo signaling pathway in humans and how the expression of different components leads to the regulation of resistance against some of the common chemo-drugs used in breast cancer treatment. The article will also discuss the chemotherapeutics that became ineffective due to the resistance and the mechanism following the process.
\end{abstract}

Keywords: drug resistance, breast cancer, chemo drugs, Hippo signaling, YAP, LATS, TAZ

\section{Introduction}

Breast cancer can be described as a fatal disease as it contributes to increasing death rates in women worldwide and is also considered the second most common type of cancer next to lung cancer. If untreated or late-diagnosed, breast cancer can easily infilter to adjoining lymph nodes and metastasize to distant significant organs such as the liver, lung, and brain. ${ }^{1}$ According to the WHO statistics, in 2020, around 2.3 million women were diagnosed with breast cancer, besides 6,85,000 deaths globally. $^{2}$ Cancer is life-threatening and remains incurable mainly because the symptoms are asymptomatic, leading to the early onset but the late prognosis. By the time cancerous cells were identified, the disease had already progressed in the patient's body. Lagging prognosis leads to the development of local and distant metastasis depending on the nature of the tumorous growth.

Chemotherapy is one of the foremost and influential ways to treat breast cancer in most countries. However, it is essential to emphasize that while applying chemotherapy to the patient, the main hurdle that prevents the diminishing of cancerous growth is drug resistance. In this case, after a few treatment cycles, the patient starts to develop resistance against the supplied drug, thus limiting the patient's benefits. ${ }^{3}$

Through the various studies conducted to modulate the process of drug resistance in cancer therapy, the Hippo pathway came to light. Hippo signaling proves to be an essential aspect in imparting drug resistance, leading to ineffectiveness in
Correspondence: Sadaf; Abdulaziz A Aloliq Email sadaf924@gmail.com; aaalieky@qu.edu.sa 
treatment. The hippo pathway is a signaling mechanism commonly witnessed in mammals and is involved in cellular processes at the genomic level leading to alteration in gene expression. Thus, the potential of genes in Hippo Signaling can be explored in significant mechanisms leading to malignancy and drug resistance in cancer therapy. Several recent data on various solid tumors have pointed out the alteration in the Hippo pathway, suggesting a possible association in pathogenesis and drug resistance. $^{3-6}$ For example, in breast cancer, the cotranscriptional factors $Y A P$ and $T A Z$ of the hippo pathway are deregulated with increased $T A Z$ and $Y A P 1$. The raised expression of YAP1 and TAZ has been linked to Her2+, Luminal $\mathrm{A}$, and Luminal $\mathrm{B}$ and triple-negative breast cancer (TNBC). ${ }^{7}$ Furthermore, the expression of $Y A P 1$ was also noticed to be linked with a low survival rate in HER 2+ positive breast cancer patients. These potential pieces of evidence suggest significant crosstalk between the hippo pathway and the molecular biology of breast cancer. ${ }^{8,9}$ So, it is crucial to understand the fundamental mechanism to develop effective chemotherapeutic treatments. $^{10}$

\section{Hippo Signaling Pathway}

The hippo signaling pathway includes the cascade mechanism responsible for controlling the size of the organs by maintaining cell growth and apoptosis in animals and humans. ${ }^{11}$ This pathway was first identified as a significant governor to regulate the organ's size and was first discovered in Drosophila melanogaster. ${ }^{12}$ This led to the discovery of the 4 tumor suppressors which are; Warts (Wts), ${ }^{12,13}$ Salvador (Sav), ${ }^{3,14}$ Hippo (Hpo $)^{15-19}$ and Mobas-tumor-suppressor (Mats). ${ }^{20}$ These tumor suppressors work by forming the core kinase cascade, which controls cell proliferation and apoptosis.

Initially, activated Hpo phosphorylates and activates the Wts-Mats complex. ${ }^{17,21}$ At the same time, Sav, which is also activated when phosphorylated by Hpo, activates the Wts-Mats complex by acting as a protein scaffold. ${ }^{17,18}$

The downstream target of the activated Wts is Yorkie (Yki) which plays the role of a transcriptional coactivator. $^{22}$ In the case of $Y k i$, the DNA binding domains are mainly absent. Despite the absence of the DNA binding domains, $Y k i$ binds to the Scalloped (Sd), a DNA binding partner, to regulate the transcription of genes. ${ }^{23}$ $W t s$ is capable of phosphorylating $Y k i$ in multiple sites, which can also be viewed as an effective regulatory mechanism to downregulate the expression of $Y k i$.
Phosphorylation at multiple sites leads to restricting the nuclear localization and cytoplasmic sequestration of $Y k i$ that transcriptionally inactivates the target gene. ${ }^{24-26}$

Meanwhile, in the absence of the signaling, Yki moves towards the nucleus and interacts with $\mathrm{Sd}$, another transcriptional factor. This results in activating the expression of diverse genes, including cyclin $E$ and diap1 (Figure 1A). The whole process results in increased expression, leading to cell proliferation and a decrease in cell apoptosis expression. ${ }^{19}$

In the case of human beings, the core elements that are involved in the mammalian Hippo pathway includes Serine/threonine kinases: Examples includes Mammalian sterile 20 like kinase1/2 (MST1/2), Large tumor suppressor1/2 (LATS1/2), Salvador Homolog 1 (SAV1), and MOB kinase activator $1 \mathrm{~A} / \mathrm{B}(\mathrm{MOB} 1 \mathrm{~A} / \mathrm{B})$.

It is important to note that $M O B \quad 1 A / B$ acts as an adapter protein for $M S T 1 / 2$ and $L A T S 1 / 2 .^{2}$

In humans, the $L A T S 1 / 2-M O B 1 A / B$ complex is phosphorylated and activated by MST1/2 - SAV1 complex, which results in the phosphorylation and inactivation of the Yes-associated protein (YAP) and co-activator TAZ, which are classified as the orthologs of the Yki in mammals. ${ }^{27}$ LATS1/2 works by phosphorylating YAP/TAZ at multiple sites and promotes its binding to 14-3-3 protein, leading to cytoplasmic retention. Phosphorylation also results in ubiquitination that directs the process of proteasomal degradation. ${ }^{27-29}$

The potential of hippo signaling is that the pathway is majorly responsible for controlling cell proliferation, apoptosis, and differentiation. ${ }^{30}$ If this pathway is altered due to the inactivation of tumor suppressor genes, the YAP-TAZ will move towards the nucleus, and once they reach the nucleus, they bind to the transcriptional factors of the TEAD family (Figure 1B) and activate the expression of genes that are responsible for promoting the cell proliferation and results in disrupted tumor growth and carcinogenesis. ${ }^{31}$

In conclusion, due to the genetic changes (gene silencing, transcriptional regulations, and mutations), the activity of the tumor suppressors is subdued, which then proceed to hyper activate the $Y A P / T A Z$ and develop different types of cancer.

\section{Cancer Progression Due to Hippo Pathway}

Hippo Pathway is primarily responsible for mediating cell proliferation, apoptosis, differentiation, and migration 
A

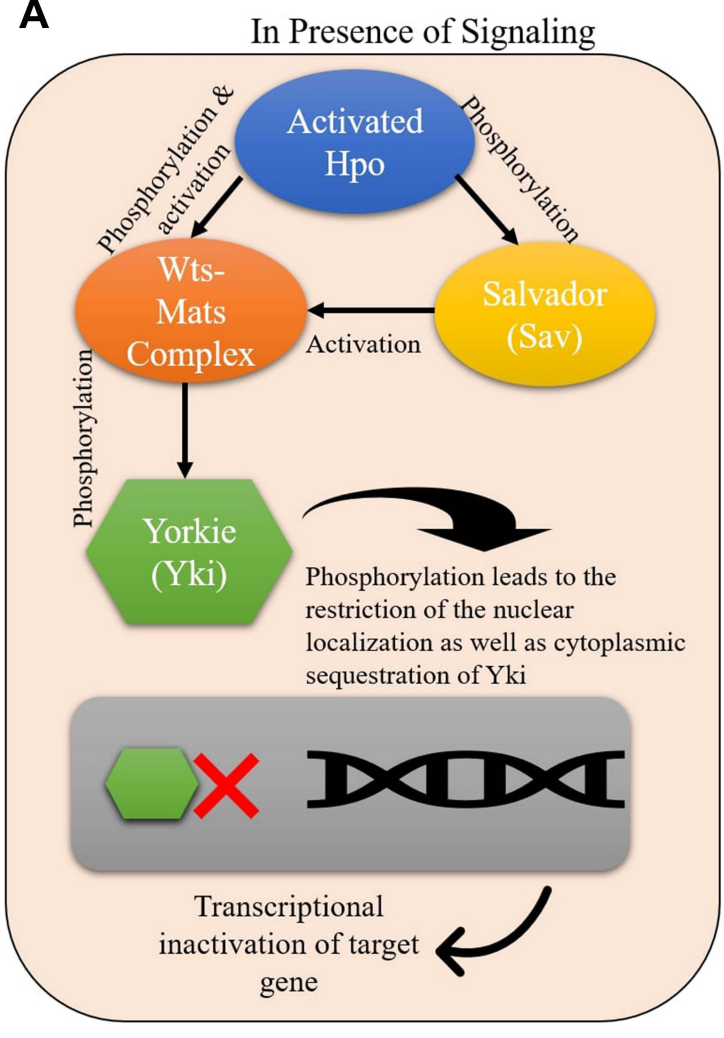

B

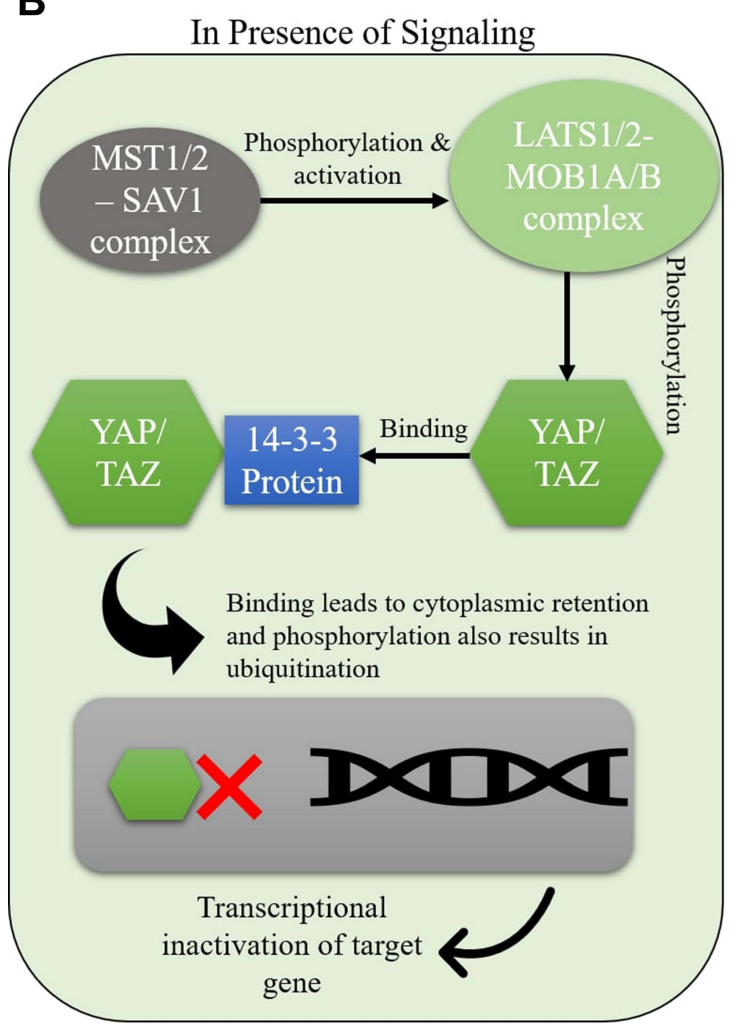

In Absence of Signaling

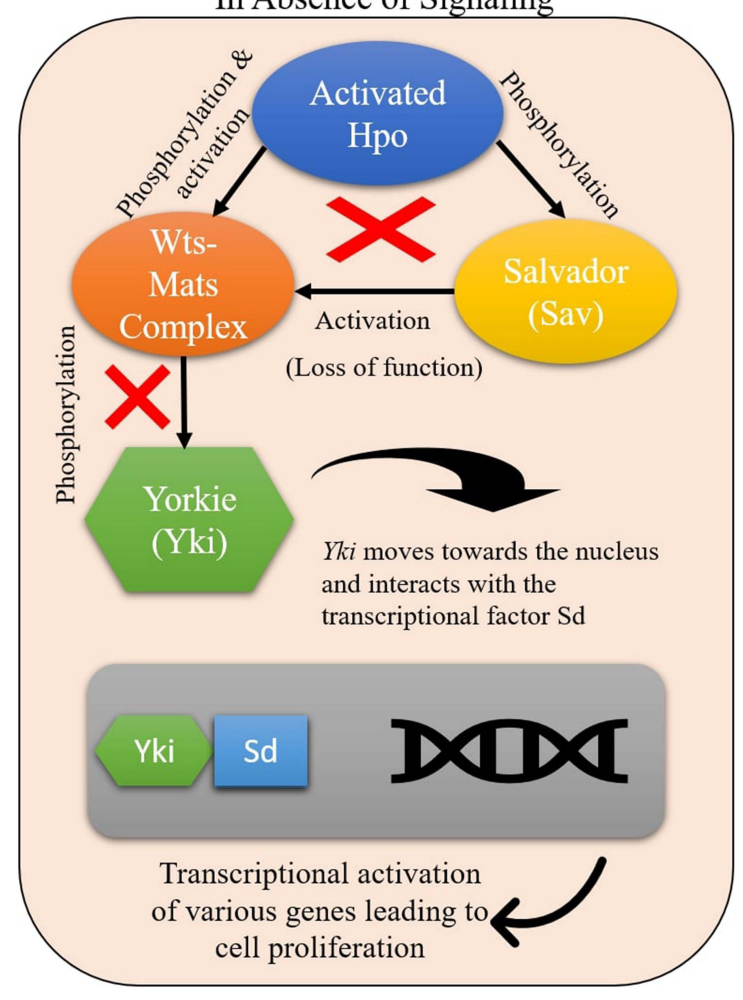

In Absence of Signaling

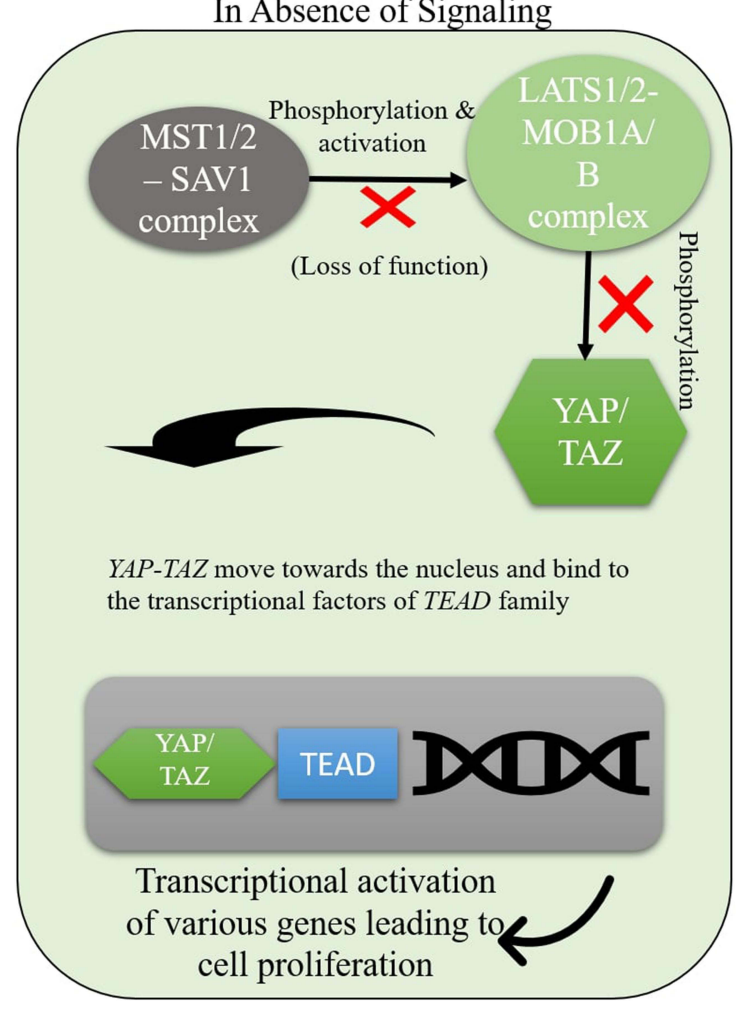

Figure I Hippo pathway gene regulation in (A) Drosophila melanogaster (B) mammals. 
(Figure 2) ${ }^{32}$ In cancer, due to the inactivation of tumor suppressors such as $L A T S 1 / 2$, the pathway is not activated, it results in the hyperactivation of the $Y A P / T A Z$, resulting in the increased expression of CRY61 and BCL2 family, which ultimately suppresses the apoptosis process as they are the factors responsible for promoting cell survival. ${ }^{26,33-35}$

In breast cancer, the upregulation of the $Y A P$ and $T A Z$ (transcriptional co-activators) are mainly responsible for cancer initiation, growth, and metastasis. TAZ plays a significant part in cancer stem cells' tumor initiation and self-renewal capacity. ${ }^{32} T A Z$, in a way, enriches cancer originating stem cells which result in the formation of tumors. ${ }^{36}$ The luminal cells even start showing the characteristics of the basal cells due to the overexpression and activity of the TAZ oncoprotein, therefore resulting in the progression of basal-like breast cancer. $^{32,36}$ YAP/TAZ mediates the activity of the other oncogenic components such as LIF and GPER. Increased expression of $Y A P$ results in the downregulation of the Leukemia Inhibitory Factor Receptor, which ultimately results in increased metastatic and invasive potential of the cells. ${ }^{37}$ The hyperactivation of YAP/TAZ results in the upregulation of the G-protein coupled estrogen receptor, which stimulates the tumor growth and movement of the cancerous cells to the surrounding tissues. ${ }^{32}$ When the Hippo pathway is not activated, the $Y A P / T A Z$ complex moves towards the nucleus. It binds to the TEAD transcriptional factors, resulting in the increased expression of many oncogenic factors such as CYR61 and CTGF, leading to progression in breast cancer. $^{34,35,38}$ The hyperactivation of YAP results in elevated levels of KLF5 proteins, thus promoting cell proliferation and cell survival (Figure 3). ${ }^{39}$

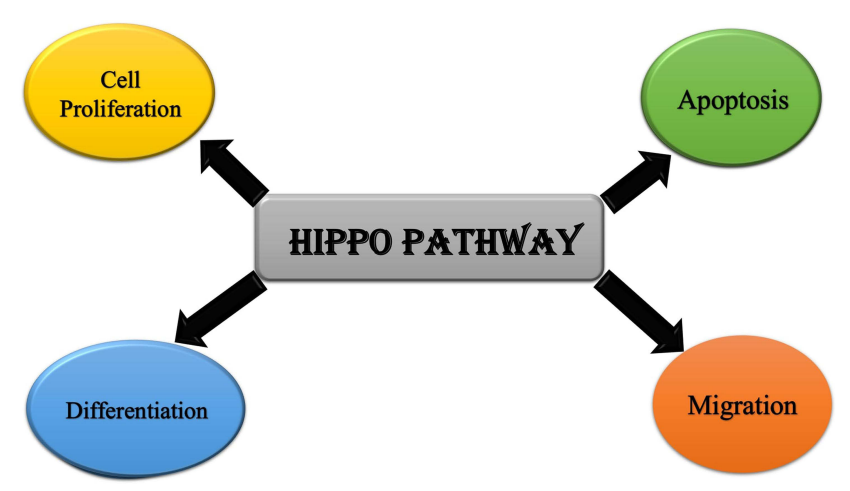

Figure 2 Cellular roles of Hippo pathway.

\section{Treatment Strategies Used in Breast Cancer}

Breast cancer belongs to the class of heterogeneous disease(s) and is basically divided into three subtypes depending on how the hormone receptors are expressed, which include estrogen and progesterone, Human epidermal growth factor receptor 2 (HER2), Triple-negative breast cancer. $^{40}$

In breast cancer, the medicinal therapies involved are mainly chemotherapy, hormonal therapy, targeted therapy, and immunotherapy.

Chemotherapy destroys the cancer cells and shrinks the tumor growth before or after surgery. Drugs mainly used in chemotherapy for breast cancer treatment are listed (Table 1). Further, these drugs can be used in combination depending upon the medical oncologist.

It is important to note that each tumor grows in a specified environment to help its growth and transmission. The targeted therapy works by targeting the specific components promoting the growth and survival of the cancerous cells, such as the specific gene, protein, or hormone. The identified targets are then treated, which results in the blockage of the growth of tumor cells as their niche is destroyed.

For example, the targeted therapies used to treat HER2-positive breast cancer are Trastuzumab, Pertuzumab, Neratinib, and Ado-trastuzumab emtansine. In addition, the therapies mentioned above are used in conjugation to chemotherapeutics, including taxanes and anthracyclines, to treat triple-negative breast cancer. ${ }^{41}$

\section{Drug Resistance Mediated by Hippo Signaling}

Although cancer largely remains incurable due to various aspects, it is essential to emphasize that chemotherapy is one of the most effective treatments for cancer patients. However, the hurdle that occurs with antibiotics is that drug resistance is also seen in cancer therapy due to genetic and epigenetic mutations and the metabolic mechanism involved in drug inactivation and efflux hampering the patients from the treatment. ${ }^{3}$ Various studies emphasized that Hippo signaling can be vital in imparting drug resistance and ineffective treatment. Bortezomib, an inhibitor that interacts with the hippo pathway, is being analyzed in the clinical trial Phase III to treat different cancers, including breast cancer. ${ }^{42}$ In an earlier study, it had been noticed that breast cancer stem-like cells 


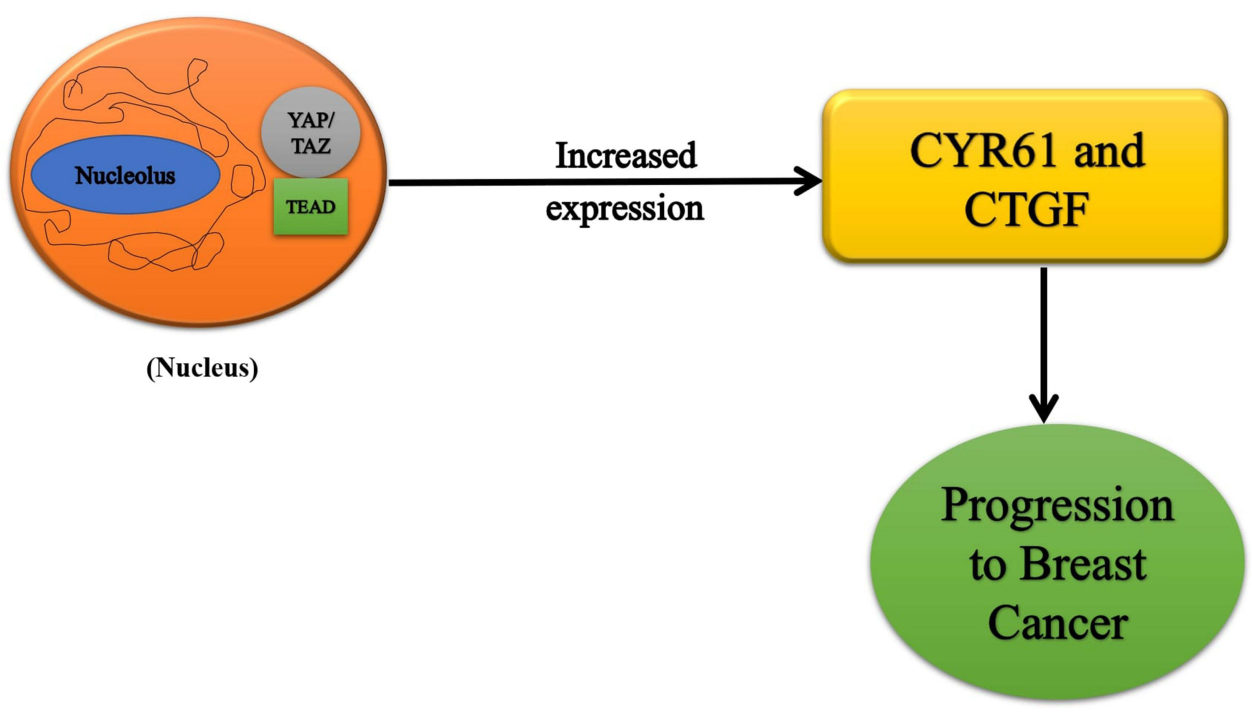

Figure 3 Hippo pathway genes in breast cancer progression.

(BCSCs), a specific group of self-renewal cells, are thought to be accountable for drug resistance and cancer spread. Interestingly, when activated, the $T A Z$ and $Y A P$ assist in maintaining breast cancer cells with stem celllike properties. ${ }^{8}$

Drug resistance is mainly observed due to the upregulation or the downregulation of the components that play an integral part in the hippo signaling pathway (Table 2).

\section{Paclitaxel}

Paclitaxel is the drug administered to treat different types of cancer and is classified as the widely used drug in this domain. The drug is plant-derived and is extracted from the Taxus brecifolia. Paclitaxel works by binding to the $\beta$ tubulin, thus maintaining the spindle formation at the time of mitosis. ${ }^{61,62}$ Resistance to paclitaxel can ascribe to the hyperactivation of $Y A P / T A Z$ or the downregulation of $h E x$. YAP can be described as a transcriptional co-activator capable of functioning as an oncoprotein by interacting and activating other transcriptional factors. ${ }^{35,63}$ For example, in the cancerous growth hyperactivation of YAP-S127A due to the abbreviated phosphorylation site, the $Y A P$ reaches the nucleus, accumulates there, and produces resistance to paclitaxel. ${ }^{52}$

$T A Z$ can be understood as an analog to YAP. Both $Y A P$ and $T A Z$ are termed oncogenes, resulting in cell proliferation and cancerous growth. ${ }^{64,65}$ Overexpression of $T A Z$ results in the development of resistance against paclitaxel as TAZ's overexpression results in the increased levels of Multidrug resistance proteins (MDR). When activated, these proteins, the cell membrane transporters, reduce the drug concentration, resulting in anti-drug resistance. ${ }^{3}$

$h E x$ is also responsible for controlling cell proliferation as it binds to the $Y k i{ }^{66}$ So, the downregulation of $h E x$ results in the increased activity of $Y k i$, resulting in the development of paclitaxel resistance (Figure 4). ${ }^{55}$ Further, Cyr61 and CTGF, the target for $T A Z / \mathrm{TEAD}$, provide resistance to paclitaxelmediated breast cancer treatment. Therefore, the activity of paclitaxel was reversed to its original form by inhibiting Cyr61 and CTGF with the short hairpin RNA method. ${ }^{45}$

\section{Lapatinib}

Lapatinib is a therapeutic drug and works as a reversible inhibitor for tyrosine kinase regions of HER2. Epidermal growth factor receptor holds significance in treating advanced or metastatic breast cancer.

It works by competing with ATP to reversibly inhibit the ATP-binding pocket by forming weak interactions. As it binds to the pocket instead of ATP, this results in the downregulation and blocking of the targeted enzymes such as Mitogen-Activated Protein Kinase and Phosphatidylinositol 3-kinase, Akt, and also results in inhibition of the mammalian target of rapamycin dependent transduction pathways, and this blocking results in the arresting the cell growth and finally results in the apoptosis of tumorous cells. ${ }^{67,68}$

It is a HER2 targeted kinase inhibitor, and it is observed that in vitro, after subsequent administration, the HER2 breast cancer cells develop resistance against the lapatinib. ${ }^{3}$ Furthermore, the association of this 
Table I Commonly Used Chemotherapeutic Drugs for Breast Cancer Treatment

\begin{tabular}{|c|c|}
\hline List of Drugs & Mode of Action* \\
\hline Paclitaxel & A plant alkaloid with anti-microtubule activity. \\
\hline Lapatinib & $\begin{array}{c}\text { Synthetic drug that ceases EGFR } \\
\text { phosphorylation. }\end{array}$ \\
\hline $\begin{array}{l}\text { Doxorubicin, } \\
\text { Epirubicin }\end{array}$ & $\begin{array}{l}\text { An anthracycline that blocks topoisomerase } \\
\text { enzyme. }\end{array}$ \\
\hline Cisplatin & Impede DNA replication. \\
\hline Tamoxifen & Compete with E2 and block its targeted role. \\
\hline 5-Flouro Uracil & $\begin{array}{l}\text { Restricts thymidine formation for DNA } \\
\text { synthesis. }\end{array}$ \\
\hline Capecitabine & Converts to 5-FU. \\
\hline Carboplatin & $\begin{array}{l}\text { Modifies the DNA molecule by forming reactive } \\
\text { platinum complexes that inhibit DNA synthesis. }\end{array}$ \\
\hline Cyclophosphamide & $\begin{array}{l}\text { Crosslinking of RNA and DNA strands leading } \\
\text { to protein synthesis inhibition. }\end{array}$ \\
\hline Eribulin & $\begin{array}{l}\text { Inhibit microtubules that are in their growth } \\
\text { phases. }\end{array}$ \\
\hline Gemcitabine & $\begin{array}{l}\text { Inhibit DNA synthesis by blocking chain } \\
\text { elongation. }\end{array}$ \\
\hline Ixabepilone & Suppressor of microtubules. \\
\hline $\begin{array}{l}\text { Methotrexate, } \\
\text { Amethoterin }\end{array}$ & $\begin{array}{l}\text { Suppress DHFR enzyme activity that leads to } \\
\text { DNA, RNA, and protein formation inhibition. }\end{array}$ \\
\hline $\begin{array}{l}\text { Protein-bound } \\
\text { paclitaxel }\end{array}$ & $\begin{array}{l}\text { Nanoparticle bound paclitaxel with the same } \\
\text { activity as paclitaxel. }\end{array}$ \\
\hline
\end{tabular}

Note: *Curated from ACCC Oncology Drug Database.

resistance development and hyperactivation of $Y A P$ and $T A Z$ has been observed in previous studies. Therefore, it is believed that the outcome of decreased/minimal
Table 2 Hippo Signaling Components and Their Associated Role in Drug-Resistant Breast Cancer

\begin{tabular}{|l|l|c|c|}
\hline $\begin{array}{l}\text { Hippo } \\
\text { Component }\end{array}$ & Dysregulation & Drugs & References \\
\hline YAP & Hyperactivation & $\begin{array}{c}\text { Lapatinib, CDK } \\
4 / 6 \text { inhibitors, } \\
\text { Paclitaxel, } \\
\text { Doxorubicin }\end{array}$ & {$[43-54]$} \\
\hline TAZ & Hyperactivation & $\begin{array}{c}\text { Paclitaxel, } \\
\text { Doxorubicin, } \\
\text { Lapatinib, CDK } \\
4 / 6 \text { inhibitors }\end{array}$ & [43-45,47,48] \\
\hline hEx & Downregulation & $\begin{array}{c}\text { Paclitaxel, Taxol } \\
{[55]}\end{array}$ \\
\hline MST & Downregulation & Cisplatin & {$[56]$} \\
\hline LATSI & Downregulation & Taxol & {$[57,58]$} \\
\hline LATS2 & Downregulation & $\begin{array}{c}\text { Doxorubicin, } \\
\text { Tamoxifen }\end{array}$ & {$[59,60]$} \\
\hline
\end{tabular}

expression of $Y A P / T A Z$ can be positively correlated with increased sensitivity of lapatinib (Figure 4). ${ }^{31}$

\section{CDK 4/6 Inhibitors}

Cyclin-dependent kinase, also known as CDKs, helps in regulating the cell cycle. CDK 4 and 6 act as a network, manage the cell cycle, and controls cell proliferation. CDK 4 and CDK 6 works as a complex with D-type cyclins, and in the case of mitogenic stimulation, the response is produced as they work together to drive cell progressions from the $\mathrm{G}$ phase to the $\mathrm{S}$ phase. ${ }^{69,70}$

CDK 4/6 inhibitors are administered to treat oncogenic growth and are administered along with hormonal therapy,

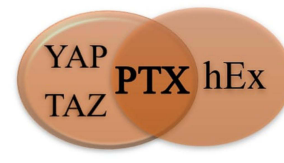

PTX : Paclitaxel
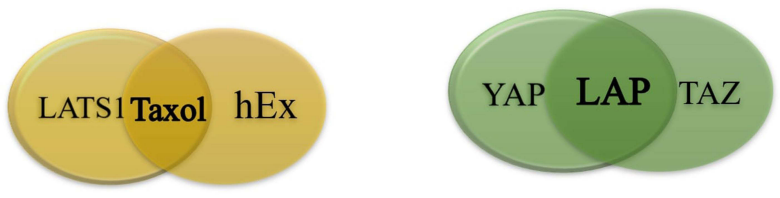

LAP : Lapatinib

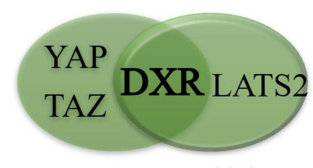

DXR : Doxorubicin

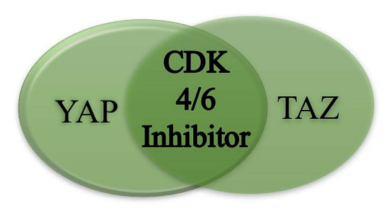

Figure 4 Hippo signaling in regulating chemo-drugs resistance. 
including the aromatase inhibitor or fulvestrant. These inhibitors are vital in treating hormone receptors, HER2, and advanced or metastatic breast cancer. ${ }^{3}$

However, their effectiveness is restricted due to the drug resistance imparted by the hyperactivation of $Y A P$ and $T A Z .{ }^{31}$ In ER-positive cases, the loss of FAT1 steered the hyperactivation of $Y A P / T A Z$ as the Hippo pathway is suppressed. As the suppression of pathway directs the increased expression of the $Y A P / T A Z$, they cluster around the CDK6 promoter and facilitate the CDK 6 transcription. Additionally, increased expression of CDK6 imparts resistance to the CDK 4/6 inhibitors in the case of the breast cancer cells and the inactivation of FAT1 protein (Figure 4). ${ }^{3}$

\section{Tamoxifen}

It is an estrogen receptor modulator, and it is utilized to medicate breast cancer. ${ }^{44}$ People diagnosed with breast cancer express the ER $\alpha$ protein and respond to ER $\alpha$ antagonists. $^{75,76}$

However, the resistance to hormone therapy is observed in patients and tamoxifen resistance is developed due to the downregulation of LATS2. ${ }^{69}$ The downregulation of LATS2 results in its co-localization with the ER $\alpha$ in the nucleus. As LATS 2 can activate the ER $\alpha$ transcription, it promotes the increased expression of $Y A P$, which in turn increases the transcription of estrogen receptor alpha, causing inhibition of the action of tamoxifen drug. ${ }^{57,60}$ Consequently, regulating the expression of LATS2 protein can overcome this resistance towards tamoxifen.

\section{Doxorubicin}

Doxorubicin falls under the category of anthracycline drugs and works by binding to the enzyme topoisomerase II and blocking its action. Since this is the enzyme that the cancerous cells require to grow and divide, the binding of doxorubicin to the topoisomerase enzyme results in cell apoptosis. Therefore, this drug is commonly administered to treat various kinds of cancer. ${ }^{71}$

Doxorubicin resistance is developed due to the hyperactivation of $Y A P / T A Z$ and the decreased expression of the LATS2. In the case of YAP, its overexpression leads to the partial actuation of the MAPK (Mitogen-Activated Protein Kinase) pathway, which promotes cell proliferation. ${ }^{3}$ It is also noted that YAP and p53 are activated when the cells accompanied by doxorubicin are subjected to treatment. Hence the activation and overexpression of wildtype $Y A P$ promote the development of resistance. ${ }^{72}$ Also, p53, in a way, controls the expression of the YAP where YAP binds to the promoter of $\mathrm{p} 53$ to maintain the apoptosis, whereas p53 by binding to YAP's promoter increases its expression, thus regulating its function. ${ }^{72}$

In the case of $T A Z$, its hyperactivation leads to the activation of the interleukin-8 (IL-8), and the increased levels of MDR proteins in Ras-transformed MCF10AT1K cells leads to the development of resistance. ${ }^{3,35}$

LATS2 is responsible for maintaining the expression levels of $Y A P / T A Z$ in the Hippo Pathway by phosphorylating the YAP/TAZ component to lead to cytoplasmic retention, ubiquitination, and eventually proteasomal degradation. In the case of breast cancer, the LATS2 levels are downregulated, and due to this, it is unable to phosphorylate the YAP component, which results in hyperactivation due to overexpression of the YAP component and further leads to drug resistance in cells. ${ }^{54}$

\section{Cisplatin}

Cisplatin falls under the category of chemotherapeutic drug and holds the utility to treat diverse classes of cancer. It works by forming bonds with DNA, causing damage which results in apoptosis. ${ }^{45}$ Therefore, cisplatin drug is always administered with paclitaxel as the first-line treatment.

It is found that in cisplatin resistance breast cancer cells, downregulation of the MST protein is observed. MST is an integral part of the Hippo signaling pathway, and MST 1/2 is responsible for phosphorylating YAP and $T A Z$ and downregulating them. Through autophosphorylation, $M S T 1 / 2$ gets activated, which in turn phosphorylates downstream LATS kinase that helps make LATS further phosphorylate the $Y A P$ and $T A Z$, consequently controlling cell proliferation. ${ }^{73,74}$ Overall, the hyperactivation results in the impartment of the cisplatin resistance on the patients. $^{55}$

\section{Taxol}

Taxol is a therapeutic drug and is commonly used to treat breast cancer patients. The primary role of taxol is the induction of apoptosis to destroy cancerous growth.

Again, the $T A Z$ protein is found to be overexpressed in breast cancer cells showing resistance towards chemo-drug Taxol. Highly expressed $T A Z$ molecules move towards the nucleus and interact with the TEAD proteins. TEAD factors are responsible for promoting the various $T A Z$ functions. ${ }^{32,33}$ 
Since $T A Z$ is a transcriptional co-activator, its increased levels mark the activation of various downstream targets, resulting in the activation and increased expression of many oncogenic factors such as CYR61 and CTGF. The TEAD response elements bind to the promoters of CYR61 and $C T G F$ and support their elevated expression. This ultimately suppresses the apoptosis process as they are the factors that are responsible for promoting cell survival and proliferation and interrupt the cancer treatment (Figure 4). ${ }^{27,35-37,45}$

\section{Conclusion}

The development of drug resistance proves to be a significant setback for chemotherapy as it is the most commonly used procedure to treat cancer patients. The molecules involved in the hippo signaling pathway play a vital role in developing this resistance. The deregulation of the hippo pathway, be it the inactivation of tumor suppressor genes $L A T S 1 / 2$ or the increased expression of oncogenes $Y A P / T A Z$, results in the disrupted expressions of downstream targets causing the cancer cells to develop resistance against the anti-cancer drugs. A recent study identified the predictive utility of $Y A P$ where the choice of drug can be used according to the $Y A P$ expression pattern. In addition, the protuberant role of YAP makes it an attractive target for the synthesis of anti-cancer drugs. ${ }^{77}$

Regulating the expression of these genes can be a better approach towards modulating cancer treatment by augmenting the effects of various chemo drugs involved in the whole process. To apply the practice in clinical treatment, the hippo pathway should be extensively studied and can be an accomplishment in targeted therapies for breast cancer treatment.

\section{Acknowledgment}

The researchers would like to thank the Deanship of Scientific Research, Qassim University, for funding the publication of this project.

\section{Disclosure}

The authors report no conflicts of interest in this work.

\section{References}

1. Sun Y-S, Zhao Z, Yang Z-N, et al. Risk factors and preventions of breast cancer. Int J Biol Sci. 2017;13(11):1387. doi:10.7150/ijbs.21635

2. World Health Organization. Available from: https://www.who.int/ news-room/fact-sheets/detail/breast-cancer. Accessed December 3, 2021.
3. Zeng R, Dong J. The Hippo signaling pathway in drug resistance in cancer. Cancers. 2021;13(2):318. doi:10.3390/cancers 13020318

4. Kyriazoglou A, Liontos M, Zakopoulou R, et al. The role of the hippo pathway in breast cancer carcinogenesis, prognosis, and treatment: a systematic review. Breast Care. 2021;16(1):6-15. doi: $10.1159 / 000507538$

5. Varelas X. The Hippo pathway effectors TAZ and YAP in development, homeostasis and disease. Development. 2014;141 (8):1614-1626. doi:10.1242/dev.102376

6. Zhang K, Qi HX, Hu ZM, et al. YAP and TAZ Take Center Stage in Cancer. Biochemistry. 2015;54(43):6555-6566. doi:10.1021/acs. biochem.5b01014

7. Maugeri-Saccà M, Barba M, Pizzuti L, et al. The Hippo transducers TAZ and YAP in breast cancer: oncogenic activities and clinical implications. Expert Rev Mol Med. 2015;17:e14. doi:10.1017/ erm.2015.12

8. Maugeri-Saccà M, De Maria R. Hippo pathway and breast cancer stem cells. Crit Rev Oncol Hematol. 2016;99:115-122. PMID: 26725175. doi:10.1016/j.critrevonc.2015.12.004

9. Shi P, Feng J, Chen C. Hippo pathway in mammary gland development and breast cancer. Acta Biochim Biophys Sin. 2015;47 (1):53-59. PMID: 25467757. doi:10.1093/abbs/gmu114

10. Wei C, Wang Y, Xiangqi L. The role of Hippo signal pathway in breast cancer metastasis. Onco Targets Ther. 2018;11:2185. doi:10.2147/OTT.S157058

11. Zhao B, Tumaneng K, Guan KL. The Hippo pathway in organ size control, tissue regeneration and stem cell self-renewal. Nat Cell Biol. 2011;13(8):877-883. doi:10.1038/ncb2303

12. Justice RW, Zilian O, Woods DF, et al. The Drosophila tumor suppressor gene warts encodes a homolog of human myotonic dystrophy kinase and is required for the control of cell shape and proliferation. Genes Dev. 1995;9(5):534-546. doi:10.1101/gad.9.5.534

13. Xu T, Wang W, Zhang S, et al. Identifying tumor suppressors in genetic mosaics: the Drosophila LATS gene encodes a putative protein kinase. Development. 1995;121(4):1053-1063. doi:10.1242/dev.121.4.1053

14. Tapon N, Harvey KF, Bell DW, et al. Salvador Promotes both cell cycle exit and apoptosis in Drosophila and is mutated in human cancer cell lines. Cell. 2002;110(4):467-478. doi:10.1016/S00928674(02)00824-3

15. Harvey KF, Pfleger CM, Hariharan IK. The Drosophila Mst ortholog, hippo, restricts growth and cell proliferation and promotes apoptosis. Cell. 2003;114(4):457-467. doi:10.1016/S0092-8674(03)00557-9

16. Jia J, Zhang W, Wang B, et al. The Drosophila Ste20 family kinase dMST functions as a tumor suppressor by restricting cell proliferation and promoting apoptosis. Genes Dev. 2003;17(20):2514-2519. doi:10.1101/gad.1134003

17. Wu S, Huang J, Dong J, et al. hippo encodes a Ste-20 family protein kinase that restricts cell proliferation and promotes apoptosis in conjunction with salvador and warts. Cell. 2003;114(4):445-456. doi:10.1016/S0092-8674(03)00549-X

18. Pantalacci S, Tapon N, Pierre L. The Salvador partner Hippo promotes apoptosis and cell-cycle exit in Drosophila. Nat Cell Biol. 2003;5(10):921-927. doi:10.1038/ncb1051

19. Udan RS, Kango-Singh M, Nolo R, et al. Hippo promotes proliferation arrest and apoptosis in the Salvador/Warts pathway. Nat Cell Biol. 2003;5(10):914-920.

20. Lai Z-C, Wei X, Shimizu T, et al. Control of cell proliferation and apoptosis by mob as tumor suppressor, mats. Cell. 2005;120 (5):675-685. doi:10.1016/j.cell.2004.12.036

21. Wei X, Shimizu T, Lai Z-C. Mob as tumor suppressor is activated by Hippo kinase for growth inhibition in Drosophila. EMBO J. 2007;26 (7):1772-1781. doi:10.1038/sj.emboj.7601630

22. Huang $\mathrm{J}$, Wu S, Barrera $\mathrm{J}$, et al. The Hippo signaling pathway coordinately regulates cell proliferation and apoptosis by inactivating Yorkie, the Drosophila Homolog of YAP. Cell. 2005;122(3):421-434. doi:10.1016/j.cell.2005.06.007 
23. Wu S, Liu Y, Zheng Y, et al. The TEAD/TEF family protein scalloped mediates transcriptional output of the hippo growth-regulatory pathway. Dev Cell. 2008;14(3):388-398. doi:10.1016/j.devcel.2008. 01.007

24. Oh $\mathrm{H}$, Irvine KD. In vivo analysis of Yorkie phosphorylation sites. Oncogene. 2009;28(17):1916-1927. doi:10.1038/onc.2009.43

25. Oh H, Irvine KD. In vivo regulation of Yorkie phosphorylation and localization. Development (Cambridge, England). 2008;135:10 81-1088. doi:10.1242/dev.015255

26. Dong J, Feldmann G, Huang J, et al. Elucidation of a universal size-control mechanism in Drosophila and mammals. Cell. 2007;130(6):1120-1133. doi:10.1016/j.cell.2007.07.019

27. Zheng Y, Pan D. The Hippo signaling pathway in development and disease. Dev Cell. 2019;50(3):264-282. doi:10.1016/j.devcel.2019. 06.003

28. Zhao B, Li L, Tumaneng K, et al. A coordinated phosphorylation by Lats and CK1 regulates YAP stability through SCF $\beta$-TRCP. Genes Dev. 2010;24(1):72-85. doi:10.1101/gad.1843810

29. Liu C-Y, Zha Z-Y, Zhou X, et al. The hippo tumor pathway promotes $T A Z$ degradation by phosphorylating a phosphodegron and recruiting the SCF $\beta$-TrCP E3 ligase. J Biol Chem. 2010;285(48):37159-37169. doi:10.1074/jbc.M110.152942

30. Hoa L, Kulaberoglu Y, Gundogdu R, et al. The characterization of LATS2 kinase regulation in Hippo-YAP signaling. Cell Signal. 2016;28(5):488-497. doi:10.1016/j.cellsig.2016.02.012

31. Atkins M, Potier D, Romanelli L, et al. An ectopic network of transcription factors regulated by hippo signaling drives growth and invasion of a malignant tumor model. Curr Biol. 2016;26 (16):2101-2113.

32. Lin KC, Park HW, Guan K-L. Deregulation and therapeutic potential of the hippo pathway in Cancer. Ann Rev Cancer Biol. 2018;2:59-79. doi:10.1146/annurev-cancerbio-030617-050202

33. Rosenbluh J, Nijhawan D, Cox A, et al. $\beta$-Catenin-driven cancers require a $Y A P 1$ transcriptional complex for survival and tumorigenesis. Cell. 2012;151(7):1457-1473. doi:10.1016/j.cell.2012.11.026

34. Zhang $\mathrm{H}$, Liu CY, Zha ZY, et al. TEAD transcription factors mediate the function of $T A Z$ in cell growth and epithelial-mesenchymal transition. J Biol Chem. 2009;284(20):13355-13362. doi:10.1074/ jbc.M900843200

35. Zhao B, Ye X, Yu J, et al. TEAD mediates YAP-dependent gene induction and growth control. Genes Dev. 2008;22(14):1962-1971.

36. Skibinski A, Breindel J, Prat A, et al. The Hippo transducer TAZ interacts with the SWI/SNF complex to regulate breast epithelial lineage commitment. Cell Rep. 2014;6(6):1059-1072. doi:10.1016/j. celrep.2014.02.038

37. Chen D, Sun Y, Wei Y, et al. LIFR is a breast cancer metastasis suppressor upstream of the Hippo-YAP pathway and a prognostic marker. Nat Med. 2012;18(10):1511-1517. doi:10. $1038 / \mathrm{nm} .2940$

38. Hiemer SE, Szymaniak AD, Varelas X. The transcriptional regulators $T A Z$ and $Y A P$ direct transforming growth factor $\beta$-induced tumorigenic phenotypes in breast cancer cells*. J Biol Chem. 2014;289 (19):13461-13474.3461-13474. doi:10.1074/jbc.M113.529115

39. Zhi X, Zhao D, Zhou Z, et al. YAP promotes breast cell proliferation and survival partially through stabilizing the KLF5 transcription factor. Am J Pathol. 2012;180(6):2452-2461. doi:10.1016/j.ajpath.20 12.02 .025

40. Guiu S, Michiels S, André F, et al. Molecular subclasses of breast cancer: how do we define them? The IMPAKT 2012 Working Group Statement. Ann Oncol. 2012;23(12):2997-3006. doi:10.1093/annonc/ mds586

41. Martin HL, Smith L, Tomlinson DC. Multidrug-resistant breast cancer: current perspectives. Breast Cancer. 2014;6:1.

42. Ye S, Eisinger-Mathason TS. Targeting the Hippo pathway: clinical implications and therapeutics. Pharmacol Res. 2016;103:270-278. doi:10.1016/j.phrs.2015.11.025
43. Lin C-H, Pelissier FA, Zhang $\mathrm{H}$, et al. Microenvironment rigidity modulates responses to the HER2 receptor tyrosine kinase inhibitor lapatinib via $Y A P$ and $T A Z$ transcription factors.". Mol Biol Cell. 2015;26(22):3946-3953. doi:10.1091/mbc.E15-07-0456

44. Li Z, Razavi P, Li Q, et al. Loss of the FAT1 tumor suppressor promotes resistance to CDK4/6 inhibitors via the hippo pathway. Cancer Cell. 2018;34(6):893-905. doi:10.1016/j.ccell.2018.11. 006

45. Lai D, Ho KC, Hao Y, et al. Taxol resistance in breast cancer cells is mediated by the hippo pathway component $T A Z$ and its downstream transcriptional targets Cyr61 and CTGF. Cancer Res. 2011;71 (7):2728-2738.

46. Zhao Y, Yang X. Regulation of sensitivity of tumor cells to antitubulin drugs by Cdk1-TAZ signaling. Oncotarget. 2015;6(26):21906. doi:10.18632/oncotarget.4259

47. Bartucci M, Dattilo R, Moriconi C, et al. TAZ is required for metastatic activity and chemoresistance of breast cancer stem cells. Oncogene. 2015;34(6):681-690.

48. Cordenonsi M, Zanconato F, Azzolin L, et al. The Hippo transducer $T A Z$ confers cancer stem cell-related traits on breast cancer cells. Cell. 2011;147(4):759-772. doi:10.1016/j.cell.2011.09.048

49. Jeong W, Kim SB, Sohn BH, et al. Activation of $Y A P 1$ is associated with poor prognosis and response to taxanes in ovarian cancer. Anti Cancer Res. 2014;34(2):811-817.

50. Xia Y, Chang T, Wang Y, et al. YAP promotes ovarian cancer cell tumorigenesis and is indicative of a poor prognosis for ovarian cancer patients. PLoS One. 2014;9(3):e91770. doi:10.1371/journal.pone.0091770

51. Zhang X, George J, Deb S, et al. The Hippo pathway transcriptional co-activator, $Y A P$, is an ovarian cancer oncogene. Oncogene. 2011;30 (25):2810-2822. doi:10.1038/onc.2011.8

52. Huo X, Zhang QI, Liu AM, et al. Overexpression of Yes-associated protein confers doxorubicin resistance in hepatocellullar carcinoma. Oncol Rep. 2013;29(2):840-846. doi:10.3892/or.2012.2176

53. Jiang $\mathrm{N}$, Hjorth-Jensen $\mathrm{K}$, Hekmat $\mathrm{O}$, et al. In vivo quantitative phosphoproteomic profiling identifies novel regulators of castration-resistant prostate cancer growth. Oncogene. 2015;34 (21):2764-2776. doi:10.1038/onc.2014.206

54. Huang J-M, Nagatomo I, Suzuki E, et al. YAP modifies cancer cell sensitivity to EGFR and survivin inhibitors and is negatively regulated by the non-receptor type protein tyrosine phosphatase 14. Oncogene. 2013;32(17):2220-2229. doi:10.1038/onc.2012. 231

55. Visser-Grieve S, Hao Y, Yang X. Human homolog of Drosophila expanded, hEx, functions as a putative tumor suppressor in human cancer cell lines independently of the Hippo pathway. Oncogene. 2012;31(9):1189-1195. doi:10.1038/onc.2011.318

56. Ren A, Yan G, You B, et al. Down-regulation of mammalian sterile 20-Like Kinase 1 by heat shock protein 70 mediates cisplatin resistance in prostate cancer cells. Cancer Res. 2008;68(7):2266-2274. doi:10.1158/0008-5472.CAN-07-6248

57. Visser S, Yang X. LATS tumor suppressor: a new governor of cellular homeostasis. Cell Cycle. 2010;9(19):3892-3903. doi:10.4161/cc.9. 19.13386

58. Ji D, Deeds SL, Weinstein EJ. A screen of shRNAs targeting tumor suppressor genes to identify factors involved in A549 paclitaxel sensitivity. Oncol Rep. 2007;18(6):1499-1505.

59. Kawahara M, Hori T, Chonabayashi K, et al. Kpm/LATS2 is linked to chemosensitivity of leukemic cells through the stabilization of $\mathrm{p} 73$. Blood. 2008;112(9):3856-3866.

60. Lit LC, Scott S, Zhang H, et al. LATS2 is a modulator of estrogen receptor alpha. Anti Cancer Res. 2013;33(1):53-63.

61. Horwitz SB. "Taxol (paclitaxel): mechanisms of action." Annals of oncology: official. J Eur Soc Med Oncol. 1994;5:S3-S6.

62. Risinger AL, Giles FJ, Mooberry SL. Microtubule dynamics as a target in oncology. Cancer Treat Rev. 2009;35(3):255-261. doi:10.1016/j.ctrv.2008.11.001 
63. Lamar JM, Stern P, Liu H, et al. The Hippo pathway target, YAP, promotes metastasis through its TEAD-interaction domain. Proc Natl Acad Sci. 2012;109(37):E2441-E2450. doi:10.1073/pnas.1212021109

64. Lei Q-Y, Zhang H, Zhao B, et al. TAZ promotes cell proliferation and epithelial-mesenchymal transition and is inhibited by the hippo pathway. Mol Cell Biol. 2008;28(7):2426-2436. doi:10.1128/ MCB.01874-07

65. Dasari S, Tchounwou PB. Cisplatin in cancer therapy: molecular mechanisms of action. Eur J Pharmacol. 2014;740:364-378. doi:10.1016/j.ejphar.2014.07.025

66. Zhao Y, Yang X. WWTR1 (WW domain containing transcription regulator 1). Atlas Genet Cytogenet Oncol Haematol. 2014;18 (11):849. doi:10.4267/2042/54169

67. Xia W, Mullin RJ, Keith BR, et al. Anti-tumor activity of GW572016: a dual tyrosine kinase inhibitor blocks EGF activation of EGFR/erbB2 and downstream Erk1/2 and AKT pathways. Oncogene. 2002;21(41):6255-6263. doi:10.1038/sj.onc.1205794

68. Ahn ER, Vogel CL. Dual HER2-targeted approaches in HER2-positive breast cancer. Breast Cancer Res Treat. 2012;131 (2):371-383. doi:10.1007/s10549-011-1781-y

69. Otto T, Sicinski P. Cell cycle proteins as promising targets in cancer therapy. Nat Rev Cancer. 2017;17(2):93-115. doi:10.1038/nrc.2016.138

70. Sherr CJ, Roberts JM. Living with or without cyclins and cyclin-dependent kinases. Genes Dev. 2004;18(22):2699-2711. doi:10.1101/gad.1256504
71. Available from: https://www.cancerresearchuk.org/about-cancer/can cer-in-general/treatment/cancer-drugs/drugs/doxorubicin\#: :text= Doxorubicin\%20is\%20a\%20type\%20of,combination $\% 20$ with\% 20other\%20chemotherapy\%20drugs. Accessed December 3, 2021.

72. Bai N, Zhang $\mathrm{C}$, Liang $\mathrm{N}$, et al. Yes-associated protein (YAP) increases chemosensitivity of hepatocellular carcinoma cells by modulation of p53. Cancer Biol Ther. 2013;14(6):511-520. doi:10.4161/ cbt. 24345

73. Pan D. The hippo signaling pathway in development and cancer. Dev Cell. 2010;19(4):491-505. doi:10.1016/j.devcel.2010.09.011

74. Chan EHY, Nousiainen M, Chalamalasetty RB, et al. The Ste20-like kinase Mst2 activates the human large tumor suppressor kinase LATS1. Oncogene. 2005;24(12):2076-2086. doi:10.1038/sj. onc. 1208445

75. Wu L, Yang X. Targeting the Hippo pathway for breast cancer therapy. Cancers. 2018;10(11):422. doi:10.3390/cancers 10110422

76. Shou J, Massarweh S, Osborne CK, et al. Mechanisms of tamoxifen resistance: increased estrogen receptor-HER2/neu crosstalk in ER/ HER2-positive breast cancer. J Natl Cancer Inst. 2004;96 (12):926-935. doi:10.1093/jnci/djh166

77. Kim HB, Myung SJ. Clinical implications of the Hippo-YAP pathway in multiple cancer contexts. BMB Rep. 2018;51(3):119-125. doi:10.5483/bmbrep.2018.51.3.018

\section{Publish your work in this journal}

Breast Cancer - Targets and Therapy is an international, peer-reviewed open access journal focusing on breast cancer research, identification of therapeutic targets and the optimal use of preventative and integrated treatment interventions to achieve improved outcomes, enhanced survival and quality of life for the cancer patient.
The manuscript management system is completely online and includes a very quick and fair peer-review system, which is all easy to use. Visit http://www.dovepress.com/testimonials.php to read real quotes from published authors. 